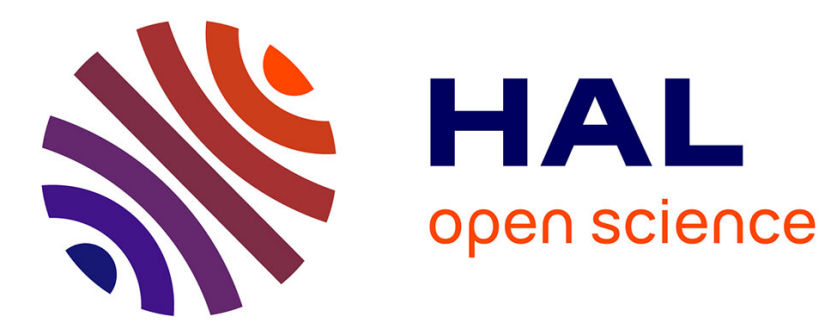

\title{
Measurement of Shear Elastic Moduli in Quasi-Incompressible Soft Solids
}

Mathieu Rénier, Jean Luc Gennisson, Christophe Barrière, Stefan Catheline, Mickaël Tanter, Daniel Royer, Mathias Fink

\section{- To cite this version:}

Mathieu Rénier, Jean Luc Gennisson, Christophe Barrière, Stefan Catheline, Mickaël Tanter, et al.. Measurement of Shear Elastic Moduli in Quasi-Incompressible Soft Solids. 18th International Symposium on Nonlinear Acoustics - ISNA 18, Jul 2008, Stockholm, Sweden. hal-03275096

\section{HAL Id: hal-03275096 \\ https://hal.science/hal-03275096}

Submitted on 30 Jun 2021

HAL is a multi-disciplinary open access archive for the deposit and dissemination of scientific research documents, whether they are published or not. The documents may come from teaching and research institutions in France or abroad, or from public or private research centers.
L'archive ouverte pluridisciplinaire HAL, est destinée au dépôt et à la diffusion de documents scientifiques de niveau recherche, publiés ou non, émanant des établissements d'enseignement et de recherche français ou étrangers, des laboratoires publics ou privés. 


\title{
Measurement of Shear Elastic Moduli in Quasi- Incompressible Soft Solids
}

\author{
Mathieu Rénier, Jean-Luc Gennisson, Christophe Barrière, Stefan \\ Catheline, Mickaël Tanter, Daniel Royer, and Mathias Fink.
}

\author{
Laboratoire Ondes et Acoustique, ESPCI, CNRS, Université Paris 7, INSERM 10 rue Vauquelin \\ 75231Paris Cedex 05, France.
}

\begin{abstract}
Recently a nonlinear equation describing the plane shear wave propagation in isotropic quasi-incompressible media has been developed using a new expression of the strain energy density, as a function of the second, third and fourth order shear elastic constants (respectively $\mu, A, D$ ) [1]. In such a case, the shear nonlinearity parameter $\beta_{S}$ depends only from these last coefficients. To date, no measurement of the parameter $D$ have been carried out in soft solids. Using a set of two experiments, acoustoelasticity and finite amplitude shear waves, the shear elastic moduli up to the fourth order of soft solids are measured. Firstly, this theoretical background is applied to the acoustoelasticity theory, giving the variations of the shear wave speed as a function of the stress applied to the medium. From such variations, both linear $(\mu)$ and third order shear modulus $(A)$ are deduced in agar-gelatin phantoms. Experimentally the radiation force induced by a focused ultrasound beam is used to generate quasi-plane linear shear waves within the medium. Then the shear wave propagation is imaged with an ultrafast ultrasound scanner. Secondly, in order to give rise to finite amplitude plane shear waves, the radiation force generation technique is replaced by a vibrating plate applied at the surface of the phantoms. The propagation is also imaged using the same ultrafast scanner. From the assessment of the third harmonic amplitude, the nonlinearity parameter $\beta_{S}$ is deduced. Finally, combining these results with the acoustoelasticity experiment, the fourth order modulus $(D)$ is deduced. This set of experiments provides the characterization, up to the fourth order, of the nonlinear shear elastic moduli in quasi-incompressible soft media. Measurements of the $\boldsymbol{A}$ moduli reveal that while the behaviors of both soft solids are close from a linear point of view, the corresponding nonlinear moduli $A$ are quite different. In a $5 \%$ agar-gelatin phantom, the fourth order elastic constant $D$ is found to be $30 \pm 10 \mathrm{kPa}$.
\end{abstract}

Keywords: Nonlinear shear elastic waves, Soft Solids, Nonlinearity parameter, Acoustoelasticity, Third harmonic measurements

PACS numbers: 43.25.Dc, 43.25.Ed

\section{INTRODUCTION}

In a recent paper, shear wave propagation theory in soft solids brought new ideas in the field of elasticity theory. Under the assumption of quasi-incompressible soft solids, Hamilton et al. [1] and Zabolotskaya el al. [2] obtained a straightforward expression of the shear strain energy density: 


$$
e=\mu I_{2}+\frac{A}{3} I_{3}+D I_{2}^{2},
$$

where $I_{2}, I_{3}$ are the strain tensor invariants defined by Landau, $\mu$ is the linear shear modulus and $A$, and $D$ the third and fourth order shear elastic constants respectively.

The aim of this paper is to quantify these three parameters in agar-gelatin based phantoms (quasi-incompressible media) and particularly for the first time the fourth order coefficient in soft solids through two different nonlinear elasticity experiments: acoustoelasticity and propagation of plane finite amplitude shear waves.

\section{ASSESSMENT OF THE THIRD ORDER LANDAU MODULUS $A$ : ACOUSTOELASTICITY}

The well-known principle of acoustoelasticity in a lossless solid is based on the variations of the speed of elastic waves induced by an uniaxially applied stress. In a quasi-incompressible media, and by considering propagating plane shear waves only, the applied stress induces an elastic anisotropy resulting from nonlinear effects. Changing the amplitude of the uniaxial stress induces an effective shear modulus $\left(\mu_{\text {eff }}=\rho_{0} V_{S i j}^{2}, i, j=1,2,3\right)$ according to a linear dependence with a slope which depends on the ratio of $A$ and $\mu$. The variations of the corresponding effective shear moduli are [3]:

$$
\begin{gathered}
\rho_{0} V_{S 13}^{2}=\mu+\sigma_{22}\left(1+\frac{A}{6 \mu}\right), \\
\rho_{0} V_{S 21}^{2}=\mu-\sigma_{22} \frac{A}{12 \mu}, \\
\rho_{0} V_{S 12}^{2}=\mu-\sigma_{22}\left(1+\frac{A}{12 \mu}\right),
\end{gathered}
$$

where $V_{S}$ is the shear wave speed according to axes of propagation presented on figure $1, \sigma_{22}$ is the uniaxial stress and $\rho_{0}$ is the density.

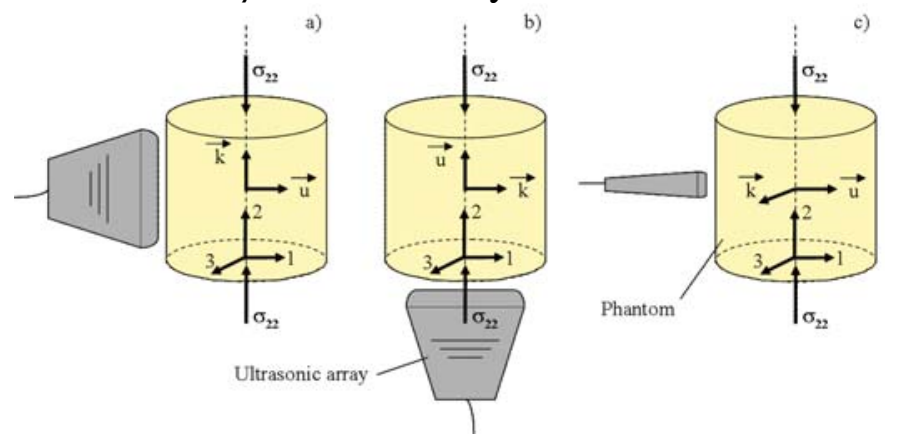

Figure 1: Acoustoelasticity experiment: The three possible configurations generating (using the acoustic radiation pressure) and detecting (with an ultrafast scanner) the propagation of polarized quasi-plane shear waves in the phantom under uniaxial stress $\left(\sigma_{22}\right)$. The three resulting shear waves are designated as [12] or [32], [21] or [23] and [13] or [31], for a), b), c) respectively.

Shear moduli $\left(\mu_{\text {eff }}\right)$ during an acoustoelasticity experiments were assessed using the supersonic shear imaging technique [4]. Using a fully programmable electronic device 
an ultrasonic beam is focused in the medium, inducing acoustic radiation force which locally generates a quasi-plane shear wave propagating perpendicularly to the direction of the ultrasonic beam (Fig. 1). Then using the same device as an ultrafast scanner (able to reach 5000 frames/s) a movie of the shear displacements is computed. From this movie, and using a time of flight algorithm, the shear wave velocity is retrieved and then the nonlinear modulus $A$.

\section{ASSESSMENT OF THE SHEAR NONLINEARITY PARAMETER: PLANE FINITE-AMPLITUDE SHEAR WAVES}

In soft solids, such as biological tissues or agar-gelatin based phantom, the very low value of the shear elasticity allows the propagation of a low frequency transverse wave $\left(f_{0}=\omega_{0} / 2 \pi=100 \mathrm{~Hz}\right)$ with a shear Mach number close to unity $\left(M_{S}=v_{0} / V_{S} \approx 0.3\right)$ [5]. These peculiar materials allow the observation of nonlinear cubic effects affecting the propagation of shear waves.

Nonlinear shear wave propagation was acquired using the same ultrafast ultrasonic scanner used for the acoustoelasticity experiments. In order to create finite amplitude plane shear waves, a vibrator bounded to a rigid plate applied on one side of the phantom was used. The modified Burgers equation for plane shear waves [5] is:

$$
\frac{\partial v}{\partial z}=\frac{\beta_{S}}{V_{S}^{3}} v^{2} \frac{\partial v}{\partial \tau}+\frac{\eta}{2 \rho c_{S}^{3}} \frac{\partial^{2} v}{\partial \tau^{2}},
$$

where $\beta_{S}=\frac{3(\mu+A / 2+D)}{2 \mu}$ is the nonlinear parameter of shear waves and $\eta$ is the shear viscosity.

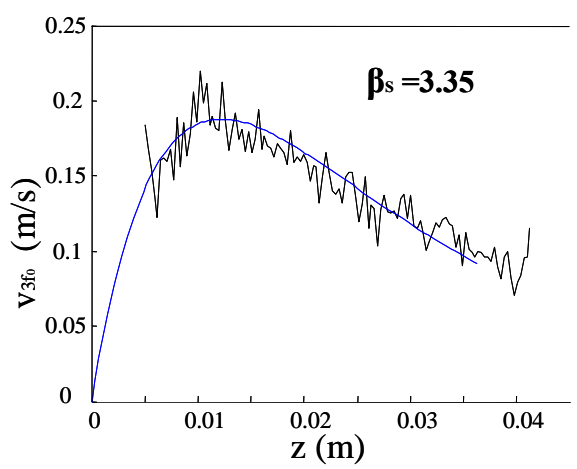

Figure 2: Absolute amplitude of the third harmonic component $v_{3 f 0}$ versus the propagation distance $z$. Measurement is fitted using Eq. 6, leading to the value of the nonlinear parameter $\beta_{\mathrm{S}}$.

For such plane waves one can observe only the generation of odd harmonics [5]. Below the shock formation distance, the amplitude of the third harmonic component can be calculated as a function of depth using a perturbation method [2]:

$$
v_{3 f_{0}}(z)=-\frac{\beta_{S} \omega_{0} v_{0}^{3}}{24 c^{3} \alpha_{0}}\left(e^{-3 \alpha_{0} z}-e^{-9 \alpha_{0} z}\right)
$$

with $\alpha_{0}$ the shear attenuation coefficient at $f_{0}$. 
The amplitude of the $3^{\text {rd }}$ harmonic is measured using a band pass filter (from 250 to $350 \mathrm{~Hz}$ ). Then, the evolution of $v_{3 f 0}$ with respect to the propagation distance $z$ is plotted for various source amplitude $v_{0}$ emissions (Fig. 2). $\beta_{S}$ is adjusted to fit the experimental data with equation 6 .

\section{RESULTS}

In acoustoelasticity experiments, the shear modulus evolution clearly exhibits the appearance of an anisotropic behavior in all phantoms. The mean value of $\mu$ and $A$ and there standard deviation were calculated from three independent values of $A$ for each direction of propagation (Table 1). One can notice that both the linear shear modulus $\mu$ and the third order elastic constant $A$ increase with the gelatin concentration of the phantom.

Table 1: Linear and second order shear moduli in agar-gelatin based phantom

\begin{tabular}{|c|c|c|}
\hline $\begin{array}{c}\text { Materials: agar-gelatin } \\
\text { phantom with in any cases } \\
\text { 3\% agar concentration. }\end{array}$ & $\begin{array}{c}\text { Linear shear modulus }(\mu \text { in } \\
\mathrm{kPa}) \text { (mean value over the } \\
\text { three measurements on each } \\
\text { axis of propagation at } 0 \\
\text { stress) }\end{array}$ & $\begin{array}{c}\text { Nonlinear shear modulus } \\
(A \text { in } \mathrm{kPa}) \text { (mean value } \\
\text { over the three } \\
\text { measurements on each } \\
\text { axis of propagation) }\end{array}$ \\
\hline$\# 1$ (5\% gelatin) & $6.6 \pm 0.6$ & $-37.7 \pm 9.8$ \\
\hline$\# 2$ (7\% gelatin) & $8.5 \pm 0.8$ & $-22.7 \pm 2.5$ \\
\hline \# 5 (8.5\% gelatin) & $9.9 \pm 0.5$ & $-5.9 \pm 1.2$ \\
\hline \# 3 (10\% gelatin) & $16.6 \pm 0.1$ & $101.4 \pm 9.0$ \\
\hline \# (13\% gelatin) & $19.2 \pm 0.1$ & $394.4 \pm 77.2$ \\
\hline
\end{tabular}

Finite amplitude wave experiment presented on Fig. 2 was realized in AG phantom \#1. The estimation of parameter $\beta_{S}=4 \pm 0.5$ allows; using the expression of $\beta_{S}$, to retrieved the fourth order nonlinear shear coefficient $D$. This is the first time that $D$ coefficient is measured in soft solid and is found to be equal to $30 \pm 10 \mathrm{kPa}$.

\section{CONCLUSION}

In this paper we have shown two possible ways to quantify the nonlinear parameters of soft solids and particularly of biological soft tissues. Furthermore, for the first time the nonlinear fourth order shear elastic coefficient is estimated. At last, further analysis must be realized on biological tissues to see if nonlinearity could have an impact on tissue characterization.

\section{REFERENCES}

[1] M. F. Hamilton et al., "Separation of compressibility and shear deformation in the elastic energy density (L)”, $J$. Acoust. Soc. Am. 116, 41-44 (2004).

[2] E. A. Zabolotskaya et al., "Modeling of non linear shear waves in soft solids”, J. Acoust. Soc. Am. 116, 28072813 (2004).

[3] J.-L. Gennisson et al., "Acoustoelasticity in incompressible media: assessment of the nonlinear shear modulus with the acoustic radiation force.”, J. Acoust. Soc. Am., 122, (2007).

[4] J. Bercoff et al., "Supersonic shear imaging: a new technique for soft tissues elasticity mapping”, IEEE Trans. Ultrason., Ferroelec., Freq. Contr., 51, 374-409 (2004)

[5] S. Catheline et al., "Observation of shock transverse waves in elastic media.”, Phys. Rev. Let. 91, 43011-43014 (2003). 